\title{
1 Formation of Microcapsules by Ultrasound Stimulation for Use 2 in Remote-controlled Drug-eluting Stents
}

3

4 Authors

5

6

9

10 Corresponding Author

11 Wei Yao

12 Wolfson Centre

13 Department of Biomedical Engineering

14 University of strathclyde

15 Glasgow G4 0NW

16 UK

17 Email:w.yao@strath.ac.uk

Word count: 5000
0 


\section{Abstract}

27 Coronary Heart Disease (CHD) is the leading cause of death globally. The placement of drug-eluting

1
stents (DESs) in diseased coronary arteries is the most successful minimally-invasive intervention to treat CHD. The key limitations of such interventional therapy are the risk of in-stent restenosis (ISR) and late stent thrombosis. This paper investigates a new drug-release system by formatting nanoparticles as drug carriers, which are later subjected to an external ultrasonic stimulus for controlled drug release remotely for DESs. The drug delivery could delay smooth muscle cell growth whilst enabling effective regeneration of a functional endothelium. Microcapsules were produced by employing a layer-by-layer technique, encapsulated with Rhodamine 6G dye used in place of antirestenotic drugs. Gold nanoparticles were employed as a shell in the microcapsules. The presence of gold nanoparticles significantly enhanced the efficiency of the ultrasonically induced dye release from the microcapsules and increased the sensitivity of the microcapsules to ultrasonic stimulation compared to those without gold nanoparticles.

Keywords: Drug-Eluting Stent, Remote Control, Nano-particle 


\section{Introduction}

Coronary Heart Disease (CHD) is a global health challenge, resulting in around 17.3 million deaths annually [1]. The placement of stents in diseased coronary arteries is the most successful minimallyinvasive intervention to treat CHD. The most advanced stents are drug-eluting stents (DES), which release a drug to inhibit the excessive smooth muscle proliferative process responsible for the inconsistent results achieved with bare metal stents. However, there is evidence that DES prevent regrowth of the endothelial cell layer (endothelium) that lines the innermost layer of the artery and it is increasingly clear that regeneration of this layer is crucial to securing positive long term outcomes [2]. There is thus a need for a stent that inhibits smooth muscle cell growth whilst enabling effective regeneration of a functional endothelium. Current DES drug release profiles lead to high initial antiproliferative drug concentrations within the artery wall, which slowly decay over weeks and months [3]. However, the process of endothelial regeneration is thought to occur within the first few days following bare metal stent implantation [4], whilst the excessive smooth muscle cell proliferation response is significantly delayed and occurs over weeks and months [5]. In this context, it is perhaps not surprising that existing DES have been associated with incomplete regeneration of the endothelium. Any drug delivery which could delay drug release until after the endothelium had fully healed would therefore be a significant advance. The aim of the research is to investigate ultrasound activated nanoparticles as a means of achieving this, with drug being released into the artery wall when it is most needed.

However, in-stent restenosis (ISR) remains a serious problem following implantation. The major reason for ISR is the injured arterial wall causing smooth muscle cell (SMC) proliferation and scar tissue accumulation [6]. Meanwhile, drug release happens spontaneously after implantation and often is 
uncontrolled [7]. To cope with this problem, nanocapsules combined with a drug delivery system can enable drug release in a specific site, as requirements [8-10].

Stimuli-responsive nanocapsules can release the drug in a controlled manner and the non-invasive nature of the technique has advantages in therapeutic application, such as reduced possibility of infection, avoided damage to surrounding tissues by devices. Most of the literature on nanoparticulate carrier systems is based on the employment of lipid, polymeric, different types of nanoparticulate carriers, and self-assembling carriers [10]. In general, the layer-by-layer assembly of nanocapsules shows advantages: it achieves the integration of component materials from different nature within the films, and it makes the incorporation of various biomolecules into the films. The layer-by-layer technique consists of renaturation of polyion adsorption, allowing the alternation of the terminal charge after each subsequent layer deposition. Furthermore, it achieves a defined control over the thickness, structure, mechanical characteristics and composition of assembled materials [11].

Gold nanoparticles (AuNP) have been studied for many years in applications such as cancer treatment [12]. They have high biocompatibility, ease of surface modification, facile synthesis, and tuneable optical characteristics [13]. Based on previous research, the release efficiency was investigated by coating gold nanoparticles in the microcapsules shell [14]. When subjected to ultrasound, the microcapsule wall undergoes a morphological change due to shear forces due to the ultrasonic oscillations. If the ultrasonic wavelength correlates to the microcapsule's size, it can maximise the effect. At $1 \mathrm{MHz}$, the wavelength closes to the size of PE microcapsules [15]. With the introduction of a rigid material such as the AuNP, the higher amount of the embedded nanoparticles leads to a decreased Young's modulus and microcapsule shell elasticity [16]. This can influence the fracture rate under ultrasonic triggering, making 
them more vulnerable to ultrasound. In addition, the gold nanoparticles are inert and are non-toxic, so they are a feasible material for medical applications. Moreover, the mechanical properties of gold nanoparticles also perform well making the microcapsules relative stable [17, 18].

Ultrasound is an effective external stimulation that can induce encapsulated drug delivery in vivo [19]. Therefore, ultrasound can act upon biomolecules. Ultrasound-responsive polymers for drug delivery systems have been studied in medical diagnostics and treatment [20]. Some polymeric systems that respond to ultrasound are mainly polymeric micelles, gels or other layer-by-layer (LbL) coated nanoparticles. Langer et al., have studied the release rate of incorporated components through the stimulation of ultrasound from polymers, including polylactides, biodegradable polyglycolides and ethylene-vinyl acetate copolymers [21-27]. It has been shown that ultrasonic stimulation can facilitate the permeation through some polymers with no erosion and enhance the decomposition rate in some biodegradable polymers [28][29]. Miyazaki et al. studied the ethylene-vinyl alcohol (EVAL) copolymer and insulin in diabetic rats and were able to control insulin release through the ultrasonic stimulation [30]. Receiving implants encapsulating insulin produced an ultrasonic stimulation $(1 \mathrm{MHz}, 1 \mathrm{~W} / \mathrm{cm} 2)$ and a significant decrease in the level of blood glucose was observed. The results demonstrated a rapid rate of release of insulin in the targeted region. In previous research of this group, they also demonstrated that the release rate of 5-fluorouracil from an EVAL copolymer can rise at desired times upon the ultrasonic stimulation in vivo [31]. Ultrasonic stimulation can induce the collapse of drug carriers and achieve payload release for the uptake of target cells. The site specificity can be promoted by incorporating a surface ligand on the carrier, which is able to bind to specific receptors for specific targeting [32][33]. 


\subsection{Synthesis of polylactic acid (PLA) microcapsules}

119 The work in this research is an attempt to develop a smart drug nanostructured delivery system that is 120 controllable using ultrasonic stimulation. This research focused on the polyelectrolytes like Polylactic 121 acid (PLA), Poly(allylamine hydrochloride) (PAH), and Poly(styrene sulfonate) (PSS) because of their

122 feasibility and their ability to function as a drug carrier. Rhodamine 6G, a kind of fluorescein dye, loaded in microcapsules was used as a drug surrogate in this model system for the controlled release studies.

The core of the microcapsule was made up of PLA microparticles. PLA microcapsules were achieved by using the nanoprecipitation method and this method has been introduced in previous research [34]. The main procedure to generate PLA/Rhodamine $6 \mathrm{G}$ microcapsules was to dissolve PLA in acetone and equilibrate for 12 hours at room temperature. Next, Rhodamine 6G was added to the acetone solution. Gradually, the colour changes of an acetone solution were observed from colourless to bright red due to the Rhodamine $6 \mathrm{G}$ becoming dissolved. The organic solution was stirred to allow the PLA and the Rhodamine $6 \mathrm{G}$ to become completely attached. After stirring, the organic solution was added drop by drop into distilled water containing no surfactant, with appropriate stirring for 2 hours. After injecting the organic phase into the aqueous phase, a conspecific microdispersion was obtained. The PLA microcapsules were suspended in water through centrifugation. The PLA/Rhodamine 6G capsules served as the cores of the microcapsules for the next layer-by-layer assemblies. The Rhodamine 6G dye served 136 as a tracer material in place of anti-restenotic drugs. It can be easily detected using fluorescence 137 spectroscopy. 
The layers coated on the PLA cores contain PAH polymers (positive charged), PSS polymer (negative 140 charged) and citrate stabilized gold nanoparticles (negative charged). With the opposite charge on 141 polyelectrolytes, the microcapsules can be formed and the number of layers can be varied as required. 142 Zeta potential measurement was employed to assess the layer-by-layer assembly process. In terms of the 143 remote ultrasonic stimulation process, the microcapsules suspended in liquid can be stimulated. In the 144 further clinical application, the system aims to allow the drug release from DES to occur in a controllable 145 manner.

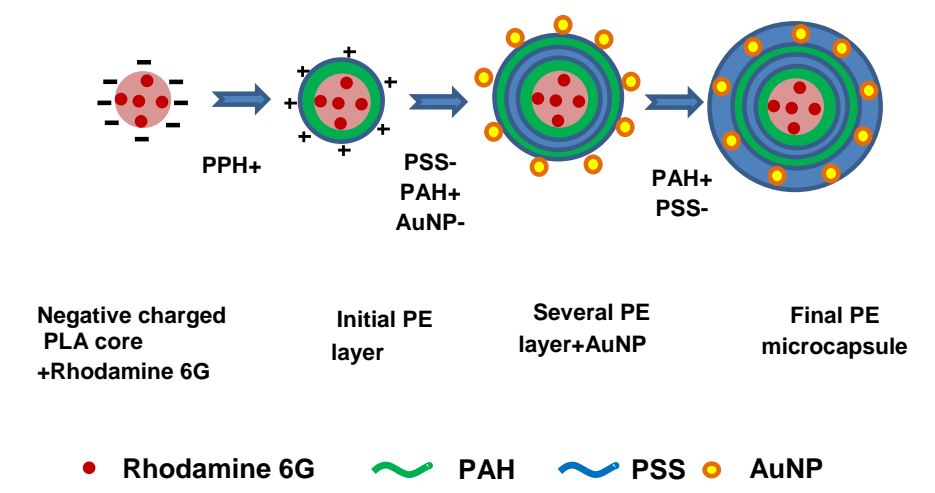

147 Figure 1 The layering processes of microocapsules.

The grey sphere represents the PLA microo-cores; the pink dots represent the Rhodamine 6G which serves as a tracer material in place of anti-restenotic drugs; the green layer represents the PAH layer which is positive charged; the blue layer represents the PSS layer which is negative charged; the gold layer represents the gold nanoparticles which are negative charged. PLA microcapsules consist of a PLA core, PAH, PSS and gold nanoparticles shells fabricated by a layer-by-layer assembly technique. In brief, 154 a $37.5 \mathrm{~mL}$ of PAH $(1 \mathrm{mg} / \mathrm{ml})$ aqueous solution was added to $25 \mathrm{~mL}$ of the PLA and stirred for 2 hours. 155 The solution was centrifuged for 15 minutes at $13000 \mathrm{rpm}$ and the supernatant was removed. The PAH coated microcapsules were resuspended in $31 \mathrm{~mL}$ of distilled water for the next layer assembly. For the 
157 second polyelectrolyte layer coating, $46.5 \mathrm{ml}$ of PSS $(1 \mathrm{mg} / \mathrm{ml})$ was added to the above solution and

158 stirred for 2 hours. After centrifuging employing the same parameters, PAH/PSS coated microcapsules

159 were re-suspended in $38 \mathrm{ml}$ of distilled water. The third layer of PAH was assembled following the same

160 process and the PLA-Rh6G/PAH/PSS/PAH microcapsules were again redispersed in $47 \mathrm{ml}$ of water.

161 Next $28.2 \mathrm{~mL}$ of citrate stabilized gold nanoparticles $(25 \mathrm{mM}$, synthesized by the sodium citrate

162 reduction method [35]), was added and was vigorously stirred for 2 hours. Following centrifugation and

163 re-suspension, the last layer of PAH was assembled in the same way. Finally PLA-Rh6G

164 /PAH/PSS/AuNP/PAH core-shell structure composite microcapsules were obtained after centrifugation 165 and re-suspension, ready for the ultrasonic stimulation experiments.

166

167

\subsection{Ultrasonic stimulation}

168

169

170

171

172

173

174

175

176

177

178

179

In this research, the trigger mechanism was remotely stimulated with ultrasound at a frequency of $1 \mathrm{MHz}$, which poses no harm for the human body within a safe range of frequency. In this research, a hand-held ultrasound device with a frequency of $1 \mathrm{MHz}$ was employed for the stimulation of PLA-Rh 6G/PAH/PSS/PAH/AuNP/PAH microcapsules. The technology of therapeutic ultrasound is a high frequency sound vibration which cannot be felt by humans, and can stimulate tissue up to $5 \mathrm{~cm}$ beneath the skin's surface. The transducer used in the experiment is a therapeutic ultrasound device ULTRALIEVE made by Actegy Ltd UK. The effective intensity that represents the amount of energy transferred to the tissues is $2.4 \mathrm{~W} / \mathrm{cm} 2$. Power supply input is at AC $100-240 \mathrm{~V} 50 / 60 \mathrm{~Hz}, 0.35 \mathrm{~A}$. The size of the transducer is $34 \mathrm{~mm}$ diameter $\times 10 \mathrm{~mm}$ high and the effective radiating area is $4 \mathrm{~cm} 2$.

It is generally tissues or microcapsules which contain components that are sensitive to the effects of heat. Abnormalities in biochemical processes may appear following the increase of temperature above the 
normal basal levels. Normally, the heat generated by ultrasound is mostly absorbed by blood circulation in vivo, and some heat is distributed through adjacent tissues. Therefore, the heat imparted onto body tissue should be given further attention, otherwise, some tissues or cells will be destroyed . The ultrasonic stimulation process in this project was not active for a long period of time to avoid overheating the microcapsules. In this project, the frequency of medical device was $1 \mathrm{MHz}$, which meets the safety requirement in human body application.

The duty cycle contains three different levels including Low (30\% duty cycle, $0.75 \mathrm{~W} / \mathrm{cm} 2$ ), Medium (40\% duty cycle, $1.0 \mathrm{~W} / \mathrm{cm} 2)$ and High $(50 \%$ duty cycle, $1.2 \mathrm{~W} / \mathrm{cm} 2)$. The duty cycle is defined as the percentage during one period in which the signal is active [36-37]. A schematic diagram of the ultrasonic stimulation imparted onto the microcapsules is shown in Figure 2.

$$
\mathrm{D}=\mathrm{T} / \mathrm{P} \times 100 \%
$$

In Equation 1, D represents duty cycle, $\mathrm{T}(\mu \mathrm{s})$ represents the duration of active signal, and $\mathrm{P}(\mu \mathrm{s})$ represents total duration of signal. For example, a 30\% duty cycle pulsed waveform would have ultrasound on for a total of $30 \%$ of the entire treatment, and off for a total of $70 \%$. A $100 \%$ duty cycle is the same of "continuous". The device used in this research, the duty cycle is fixed which includes $30 \%$, $40 \%$ and $50 \%$. 


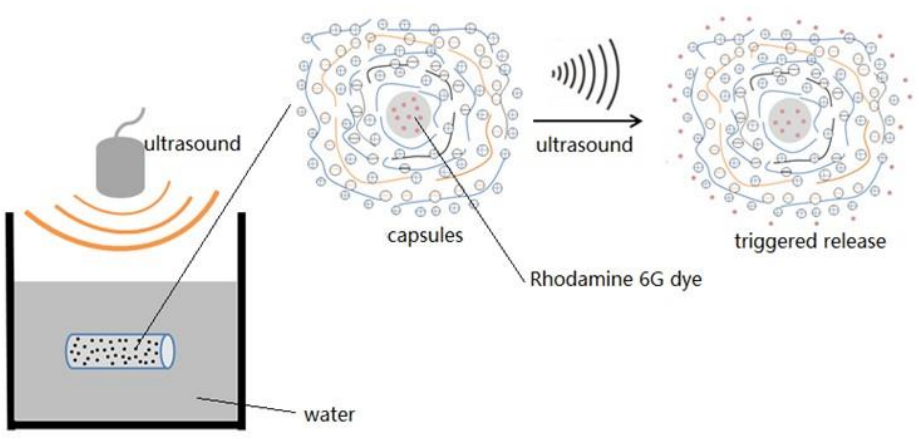

210 Figure 2 Schematic diagram of the ultrasonic stimulation imparted onto the microcapsules.

212 The PLA-Rh6G/PAH/PSS/PAH/AuNP/PAH microcapsules were suspended in the tube and the tube was 213 fixed in water. The ultrasound device was fixed and kept working, which is shown on the left of figure 2. 214 In the microcapsules details shown on the right of figure 2, the gray spheres represent the PLA 215 microcapsules, the red particles represent Rhodamine 6G dye, the blue curves represent the PAH layer 216 (positive charged), the black curves represent the PSS layer (negative charged), and the yellow curves 217 represent the AuNP shell. The dye is released by the stimulation of ultrasound.

219 In the future, these drug-loaded microcapsules will be planned to apply to the stents, allowing for a 220 stimuli-response to ultrasound.

\section{Results}

\subsection{SEM analysis microcapsules}


227 image of the microcapsules is shown in Figure 3.

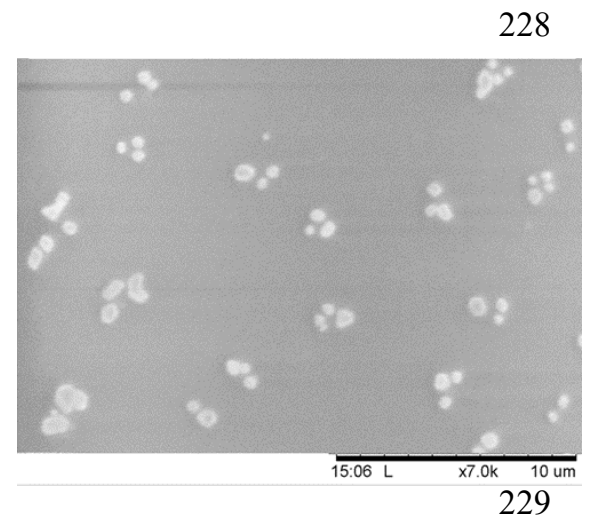

230

231

232

233

234 244 in Figure 4. (a)

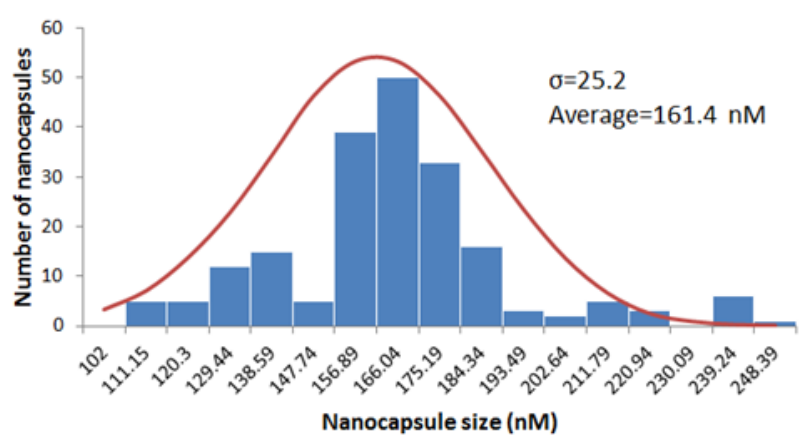

(b)

Figure 3. SEM image of PLA-Rh6G/PAH/PSS/PAH/AuNP/PAH microcapsules. (a) SEM image of PLA/Rh6G/PAH/PSS/PAH/AuNP/PAH microcapsules. (b) The size distribution of microcapsules.

Figure 3 shows that the majority of the microcapsules have dispersed spherical shapes with an average size of $165 \pm 25 \mathrm{~nm}$ ( 200 microcapsules were accounted).

\subsection{Characterisation of the microcapsule assemblies}

In this research, the microcapsules were synthesized with four polymer layers and one AuNP layer, which contained opposite charges, via layer-by-layer self-assembly processes. To examine the layer-bylayer assembly of PAH, PSS and AuNP on the PLA microcapsules, the sequential assembly procedures were monitored by means of zeta potential of the microcapsules. The variation of zeta potential with the sequential adsorption of the polyelectrolyte layer for the PAH/PSS/PAH/AuNP/PAH coatings is shown 


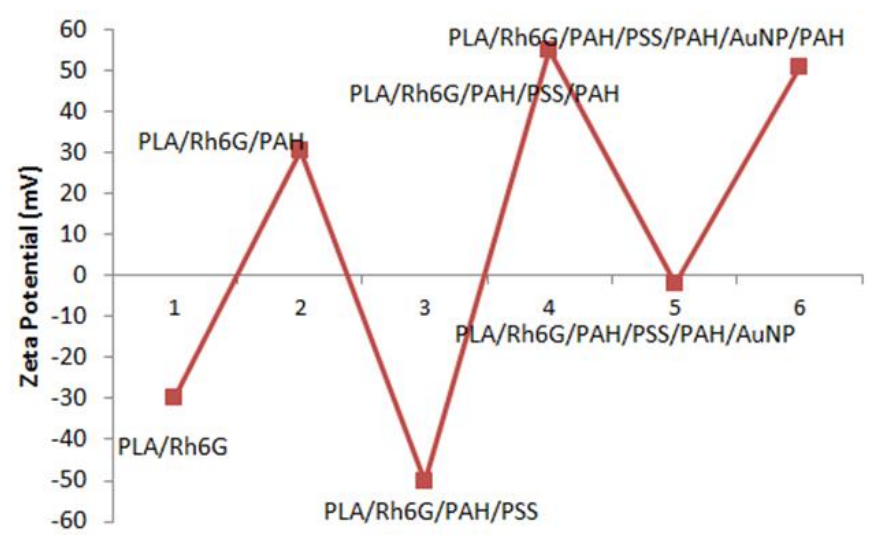

Figure 4. Zeta potential measurements in the layer-by-layer assembly of the micro-capsules.

251 The zeta potential value of the pristine PLA microcapsules was negative $(-34.5 \mathrm{mV})$. This is believed to 252 be due to the carboxylic groups present at the surface of the PLA microcapsules [38]. It was observed 253 from the zeta potential measurements that the adsorption of a positively charged PAH layer on the PLA254 Rh6G microcapsules changed the zeta potential from $-34.5 \mathrm{mV}$ to $+30.4 \mathrm{mV}$. Subsequently, the deposition of a PSS layer led to another potential reversal from +30.4 to $-50 \mathrm{mV}$. Further alternating 256 deposition of the PAH, AuNP and PAH led to continuous reversals in zeta potentials. This revealed a 257 stepwise layer assembly during the fabrication of the composite microcapsules.

259 The UV-vis spectrum of the PLA-Rh 6G/PAH/PSS/PAH/AuNP microcapsules is shown in Figure 5. An 260 absorption band due to the surface plasmon resonance of gold nanoparticles was observed indicating the 261 presence of gold nanoparticles in the microcapsules. A red-shift of the surface plasmon band from 519 $262 \mathrm{~nm}$ shown in Figure 5(b) to $556 \mathrm{~nm}$ in figure.5 (a) may possibly be due to the aggregation of the gold 263 nanoparticles when they were assembled onto the microcapsules. This absorption overlaps with a slope 
264 of scattering from the large microcapsules. The absorption of Rhodamine $6 \mathrm{G}(526 \mathrm{~nm})$ is not visible here

265 because it overlaps with the strong scattering of the micro-capsules and also the absorption band of the 266 gold nanoparticles.

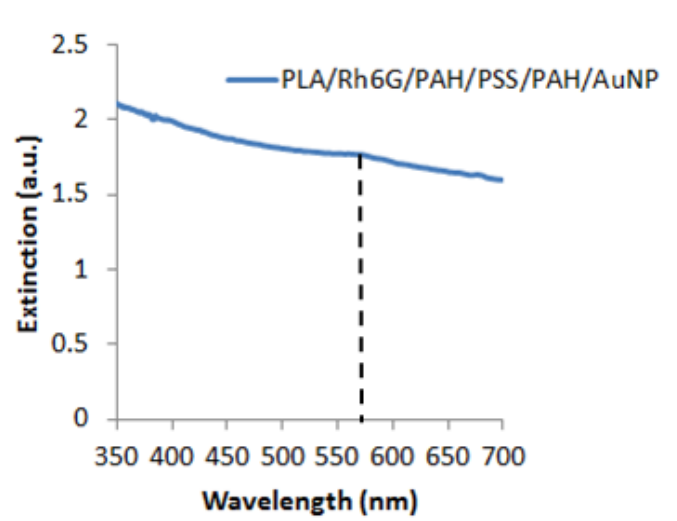

(a)

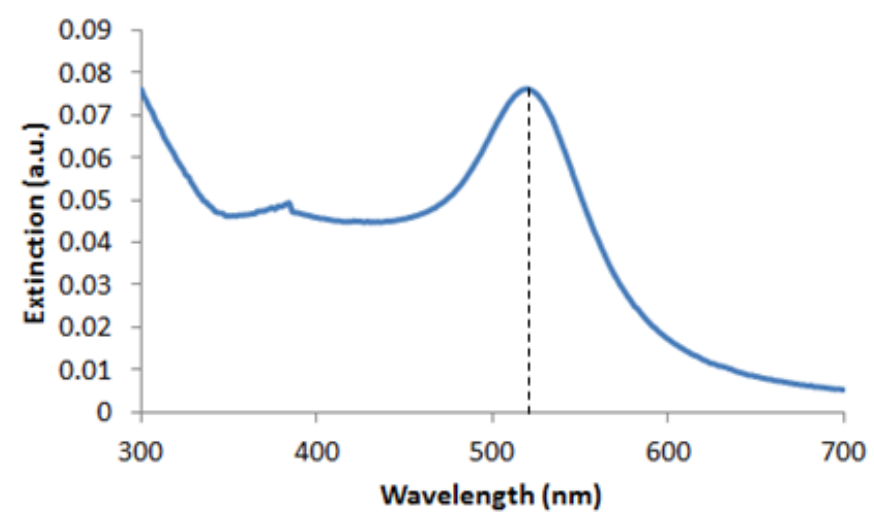

(b)

Figure 5. (a) Extinction spectrum of the PLA/Rh6G/PAH/PSS/PAH/AuNP/PAH micro-capsules (556nm). (b) UV-vis extinction spectrum of colloidal gold nanoparticles $(519 \mathrm{~nm})$.

\subsection{Ultrasonic stimulation of the microcapsules}

The release of Rhodamine $6 \mathrm{G}$ dye from the microcapsules after each ultrasonic stimulation was examined via the measurement of concentration of Rh6G in the supernatants. Both the samples and the control samples (microcapsules without gold nanoparticles) undergo ultrasonic stimulation at three different duty cycles. Figure 6 shows the fluorescence intensity change of the dye released from the PLARh6G/PAH/PSS/PAH/AuNP/PAH microcapsules against the ultrasonic stimulation times of the different duty cycles. The measurement was taken after each 15 minutes of stimulation. 


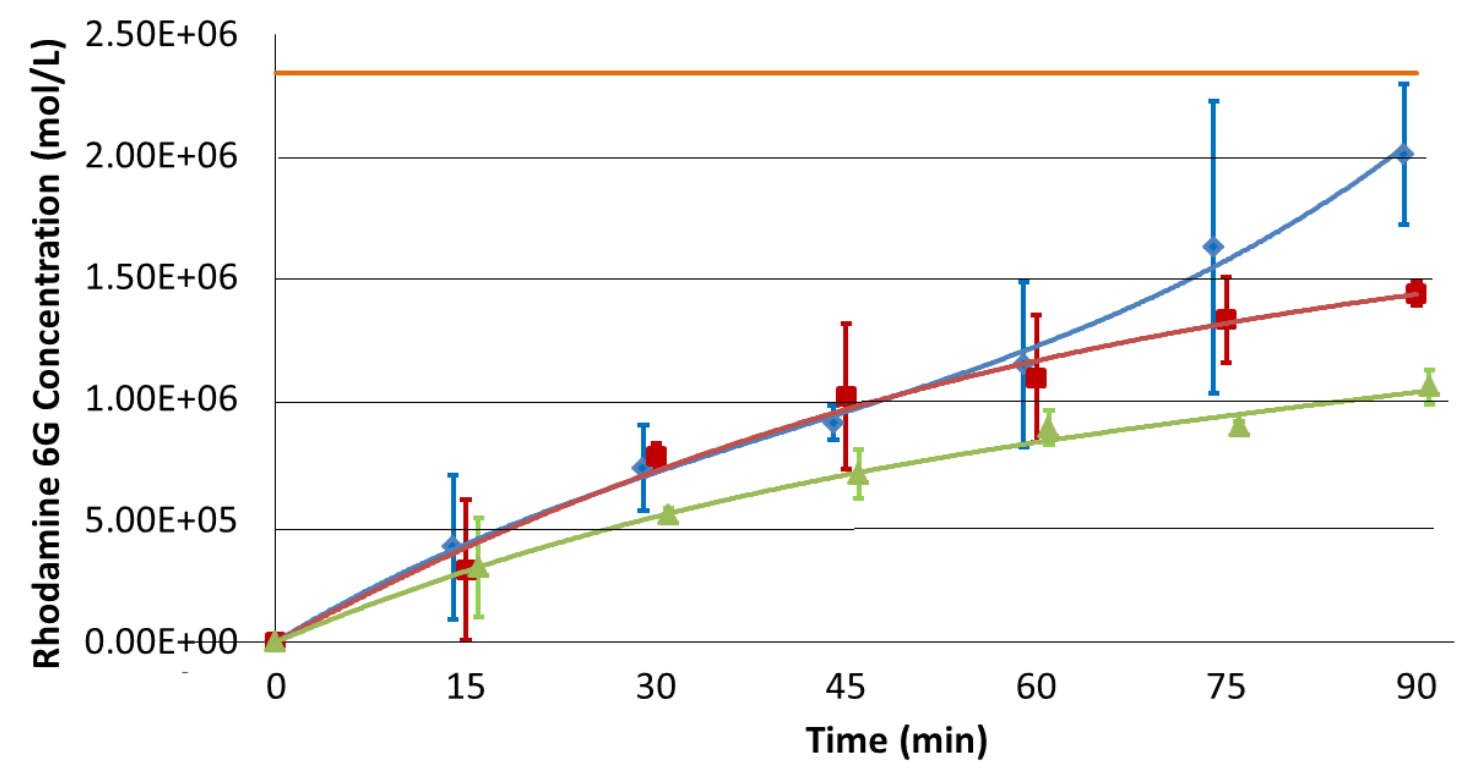

- High - Medium $\triangle$ Low — Initial dye adsorbed onto the nanocapsules

283 Figure 6. Rhodamine 6G concentration released from the capsules with gold nanoparticles against the ultrasonic stimulation 284 time of the different duty cycles. The orange line represents the total concentration of Rhodamine $6 \mathrm{G}$ contained in the 285 capsules.

287 The measurement was repeated twice independently in the collection of the presented in vitro data. The 288 graph represents means with error bars for six times. Graph markers denote the data mean. The bars 289 represent one standard deviation of the mean. Rhodamine $6 \mathrm{G}$ concentration in Figure 6 showed that 290 samples after high-duty cycle ultrasonic stimulation carried out 6 times, 15 minutes each time, released 291 more of the dye than under medium or low-duty cycle treatment. Little dye was released from these 292 microcapsules after the first 15 minutes of the stimulation. Clear increases of Rhodamine $6 \mathrm{G}$ were 293 observed after the second stimulation, although no significant differences were found among the different 294 duty cycles. With the increase of the ultrasonic stimulation times, the Rhodamine 6G concentration 295 further increased. This indicates that the ultrasonic treatment has a promoting effect on the dye release 296 from the microcapsules with the gold nanoparticles. After the fourth (total 60 minutes) ultrasonic 
treatment, the dye was still released from the samples. However, the rate of the Rhodamine 6G concentration changes was reduced, particularly for the medium and low-duty cycle treatments. After the 299 sixth ultrasonic stimulation (90 minutes), the Rhodamine 6G concentration of the dye released from the sample under the high-duty cycle treatment was close to the initial total intensity of the dye adsorbed onto the microcapsules. This implies that most of the dye contained in the samples had been released. In 302 comparison, the Rhodamine $6 \mathrm{G}$ released from the samples with the medium or low-duty cycle of 303 ultrasound was lower than with the high-duty cycle. This means that there was still some dye in the 304 microcapsules. This is not surprising as the medium or low-duty cycle of ultrasound presented lower 305 power intensities than the high-duty cycle of ultrasound, causing a lower dye to be released from the 306 microcapsules. Nevertheless, the dye released from the microcapsules with ultrasonic stimulation was 307 still presented.

To study the influence of gold nanoparticles on the efficiency of the dye release, microcapsules without 310 gold nanoparticles in their shells were also synthesized and analyzed. Their results are shown in Figure 7. 311 The graph represents means with error bars for six times. Graph markers denote the data mean. The bars 312 indicate data range.

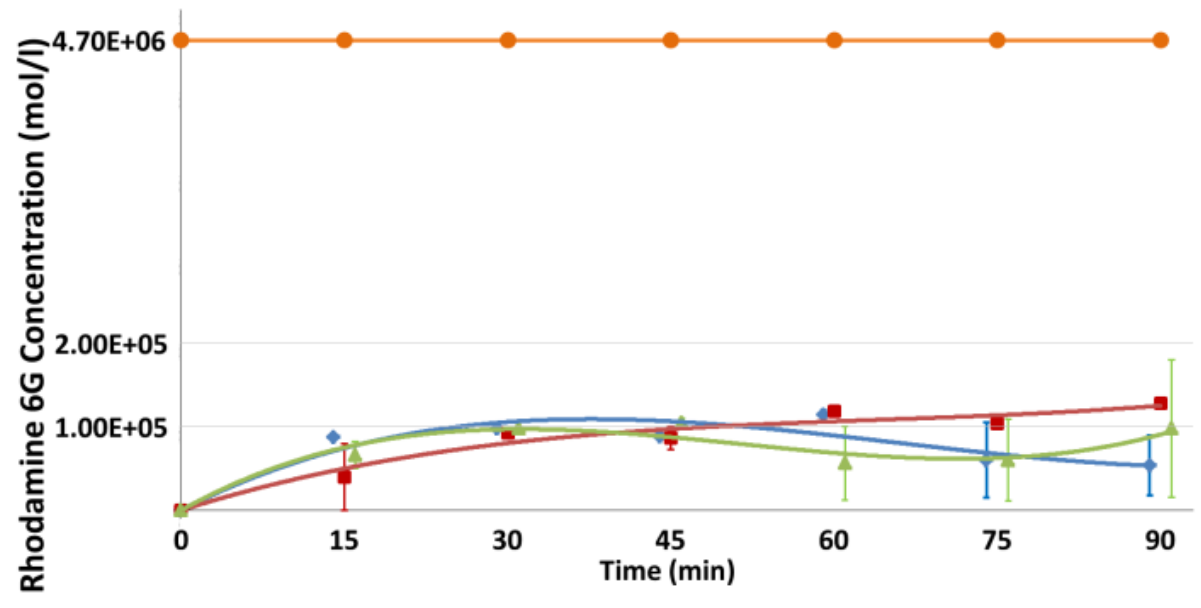

- low - Medium $\Delta$ High - Initial dye adsorbed onto the nanocapsules 
315 Figure 7. Rhodamine 6G concentration of the dye released from the capsules without gold nanoparticles measured after 316 various ultrasonic stimulation times of different duty-cycles.

318 It shows that there is no obvious changes in the concentration and R6G concentration is below $3 \%$ of the 319 total concentration after six cycles of stimulation. However, the change of fluorescence intensity with 320 the increase of the ultrasonic stimulation time was small. After the 6th stimulation, the concentration of 321 Rh6G was just $10 \%$ of the total initial concentration of Rh6G. Moreover, there was no significant 322 difference in the efficiency of the dye released by employing different duty cycles. It is thus clear that 323 gold nanoparticles in microcapsules play an important role in the ultrasonic induced release of dye.

Finally, the release of dye from samples $(1.5 \mathrm{ml})$ stored at room temperature at 200 without ultrasonic treatment during a period of four weeks was also studied as a comparison. Both microcapsules with and 327 without gold nanoparticles were used in this study. Figure 8 shows the Rhodamine $6 \mathrm{G}$ concentration 328 changes of the dye released from microcapsules after a long period of storage at room temperature at 20 329 degrees.

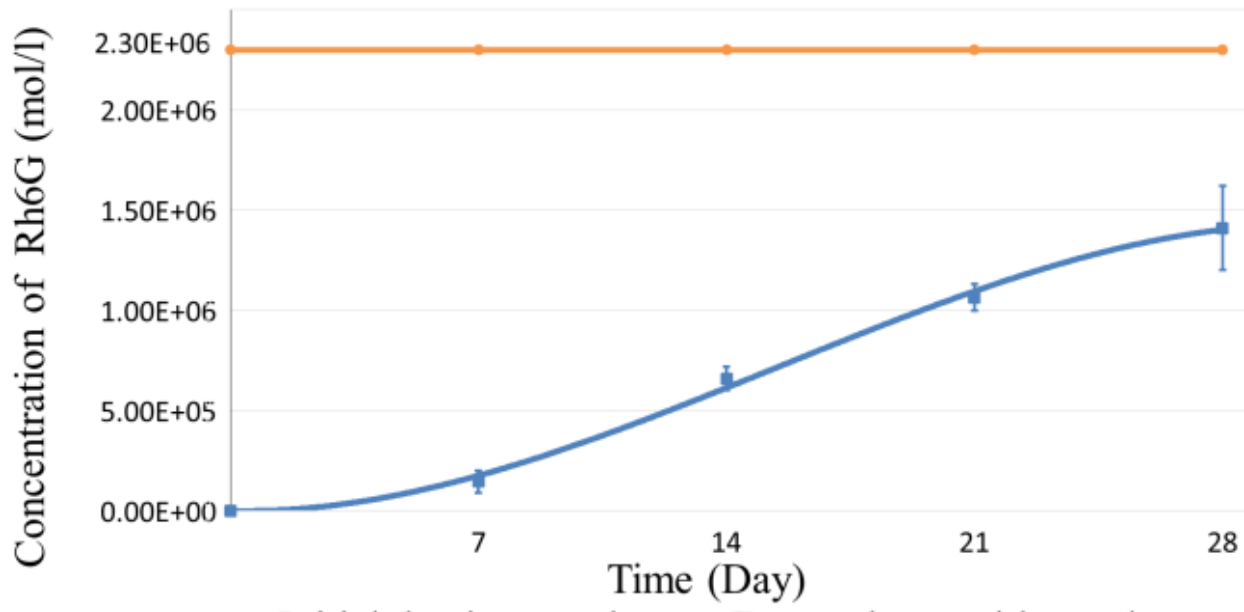




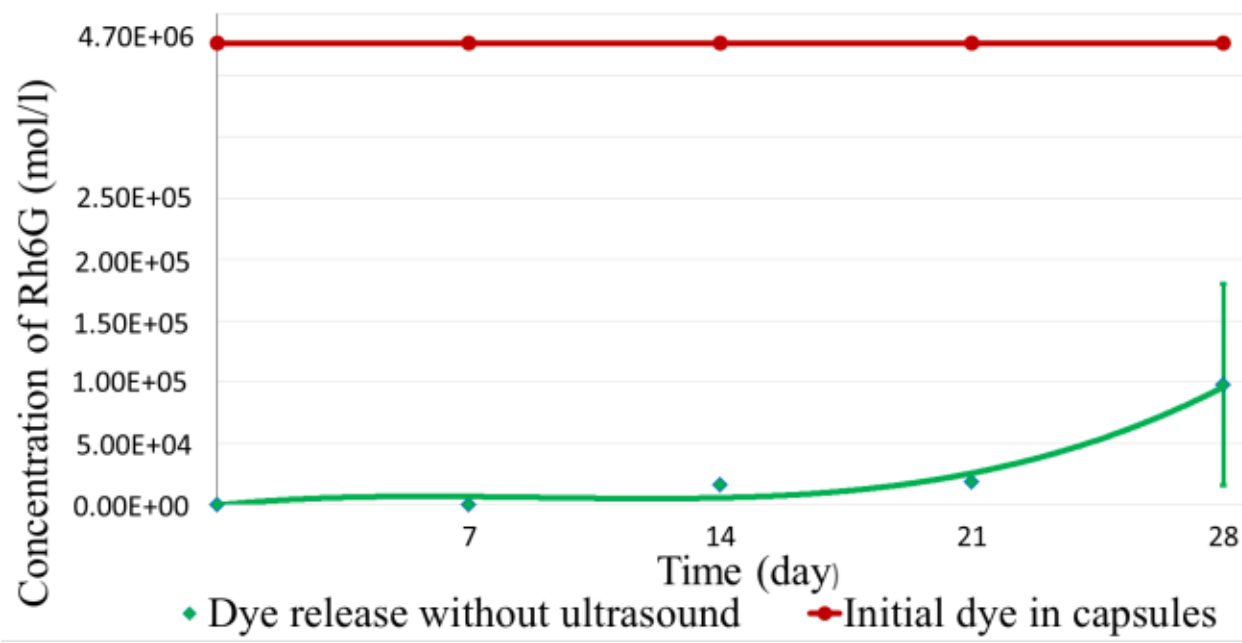

(b)

Figure 8. Rhodamine 6G concentration of dye released from capsules (a) with and (b) without gold nanoparticles after room temperature storage (without any ultrasonic stimulation). The red line represents the Rhodamine 6G contained in the samples .

Figure 8 (a) shows that after an initial small release in the first week, a large amount of dye was released from microcapsules with gold nanoparticles in the following three weeks. The graph represents means with error bars for five times. Graph markers denotes the data mean. The bars indicate data range. In contrast, no obvious changes in the Rhodamine $6 \mathrm{G}$ concentration was observed in the case of microcapsules without gold nanoparticles for the first few weeks. The intensity of released dye after 4 weeks was significantly weak compared to that from microcapsules with gold nanoparticles. This finding suggests that microcapsules containing gold nanoparticles are less stable compared to those without gold nanoparticles.

\section{Discussion}

In this study, the presence of gold nanoparticles allowed the dye to be released from PLARh6G/PAH/PSS/PAH/AuNP/PAH microcapsules more efficiently, compared to microcapsules without 
gold nanoparticles. According to these results, several factors may play a role in the stimulation process. The presence of gold nanoparticles in the microcapsules makes the shells stiffer and lower in their elasticity, which may have an effect on how easily the shells will move when applying an oscillating force to them in liquid by means of ultrasonic stimulation. If they are moved, the rupturing may occur as expected. As illustrated by Fery et al., the presence of inorganic nanoparticles increases the density contrast of a microcapsule shell, which also decreases the elasticity of the shell. These are important for achieving high efficiency in the ultrasonically treated release of compounds encapsulated in capsules [39].

The effects of ultrasound as a release trigger may be attributed to acoustic cavitation in liquids under ultrasonic vibrations with a frequency of more than $20 \mathrm{kHz}$. As demonstrated by Maria N. Antipina et al., ultrasound energy produces pressure and can cause the shrinkage of gas-filled nanobubbles. Then, the cavitating nanobubbles implode, which produces local shock waves and destroy the nanocapsules assemblies [40-44]. Even at low input power, the collapse of microbubbles in liquid results in an enormous concentration of energy. When the capsules are subjected to ultrasonic stimulation, shear forces between the successive fluid layers occurs, which leads to the disruption of the capsule assemblies and the subsequent release of their payloads [39]. Therefore, acoustic cavitation resulted in dye release from the nanocapsules in this study is a possible factor.

As for the nanocapsules without gold nanoparticles, they presented little dye release in this research. It can be assumed that the capsules may be disrupted because of the absorption of intense energy of ultrasound over a longer time. In previous research by academics, ultrasonic stimulation operating on the capsules has been proven to produce heat. High temperature causes changes in permeability of the outer 
373 shell and can even rupture the capsules. Subsequently, the release of capsules payload will be achieved

374 [45]. The heat produced by ultrasound may also have an influence on the dye released from samples 375 without gold nanoparticles.

\section{Conclusions}

378 This work aims to investigate a drug delivery system with stimuli-responsive polymeric microcapsules 379 that can achieve a locally controlled drug release by means of remote ultrasonic stimulation. The 380 microcapsules were synthesized with polyelectrolyte layers and gold nanoparticles by means of a layer381 by-layer assembly technique, encapsulated with a model dye used in place of anti-restenotic drugs. The 382 microcapsules with and without gold nanoparticles embedded in the shell were examined for their 383 response to ultrasonic stimulation. The presence of gold nanoparticles significantly enhances the 384 efficiency of the ultrasonically induced dye release from the microcapsules. It is shown that the 385 microcapsules containing gold nanoparticles are more sensitive to ultrasonic treatment compared with the 386 microcapsules without gold nanoparticles. Such a method will give the interventional cardiologist more 387 control over the medical implants.

\section{Declarations}

390 Interests conflict: None

391 Funding: None

392 Ethical approval: Not required

\section{References} $397 \quad 2011$. 
[2] Otsuka F, Finn AV, Yazdani SK, Nakano M, Kolodgie FD, Virmani R. The importance of the endothelium in atherothrombosis and coronary stenting, Nat Rev Cardiol 2012;9: 439-53.

[3] Acharya G, Park K. Mechanisms of controlled drug release from drug-eluting stents. Adv Drug Deliv Rev 2006;58: 387-401.

[4] Sprague EA, Tio F, Ahmed SH, Granada JF, Bailey SR. Impact of parallel micro-engineered stent grooves on endothelial cell migration, proliferation, and function: an in vivo correlation study of the healing response in the coronary swine model. Circ Cardiovasc Interv 2012; 5: 499-507.

[5] Steigerwald K, Ballke S, Quee SC, Byrne RA, Vorpahl M, Vogeser M, Kolodgie F, Virmani R, Joner M. Vascular healing in drug-eluting stents: differential drug-associated response of limus-eluting stents in a preclinical model of stent implantation. EuroIntervention 2012;8:752-9.

[6] Ito S, Nakasuka K, Sekimoto S, Miyata K, Inomata M, Yoshida T \& Sato K. Intracoronary Imaging and Histopathology of Late Phase In-Stent Restenosis after Coronary Stent Implantation. ISRN Vascular Medicine 2012; Article ID 678137, 11 pages. http://dx.doi.org/10.5402/2012/678137

[7] Kiran U, Makhija N. Patient with recent coronary artery stent requiring major non cardiac surgery. Indian J Anaesth 2009; 53(5): 582.

[8] Ma WJ, Yuan XB, Kang CS, Su T, Yuan XY, Pu PY, Sheng J. Evaluation of blood circulation of polysaccharide surface-decorated PLA nanoparticles. Carbohydr Polym 2008; 72(1): 75-8.

[9] Reed AM, Gilding DK. Biodegradable polymers for use in surgery_poly (glycolic)/poly (Iactic acid) homo and copolymers: 2. In vitro degradation. Polymer1981; 22(4): 494-498.

[10] Anderson JM, Shive MS. Biodegradation and biocompatibility of PLA and PLGA microspheres. Adv. Drug Deliv Rev 2012; 64:72-82.

[11] Couvreur P, Vauthier C. Nanotechnology: intelligent design to treat complex disease. Pharm Res 2006; 23(7): 1417-1450. 
421 [12] Borges J, Rodrigues LC, Reis RL, Mano JF. Layer-by-layer assembly of light-responsive polymeric 422 multilayer systems. Adv Funct Mater 2014; 24(36): 5624-564.

423 [13] Jain S, Hirst DG, O'sullivan JM. Gold nanoparticles as novel agents for cancer therapy. Br J Radiol $424 \quad 2012 ; 85(1010): 101-13$.

425 [14] Huang X, El-Sayed MA., Gold nanoparticles: optical properties and implementations in cancer 426 diagnosis and photothermal therapy. Int $\mathrm{j}$ adv res 2010; 1(1):13-28.

427 [15] Pavlov AM, Saez V, Cobley A, Graves J, Sukhorukov GB, Mason TJ. Controlled protein release 428 from microcapsules with composite shells using high frequency ultrasound-potential for in vivo 429 medical use. Soft Matter 2011; 7(9):4341-4347.

430 [16] Antipina MN, Sukhorukov GB. Remote control over guidance and release properties of composite 431 polyelectrolyte based capsules. Adv Drug Deliv Rev 2011; 63(9): 716-29.

432 [17] Skrabalak SE, Chen J, Sun Y, Lu X, Au L, Cobley CM, Xia Y. Gold nanocages: synthesis, 433 properties, and applications. Acc Chem Res 2008; 41(12): 1587-1595.

434 [18] Yu YY, Chang SS, Lee CL, Wang CC. Gold nanorods: electrochemical synthesis and optical 435 properties. J Phys Chem B 1997;101(34): 6661-6664.

436 [19] Kost J, \& Langer R. Responsive polymer systems for controlled delivery of therapeutics. Trends 437 Biotechnol 1992;10:127-131.

438 [20] Zhao YZ, Du LN, Lu CT, Jin YG, Ge SP. Potential and problems in ultrasound-responsive drug 439 delivery systems. Int J Nanomedicine 2013; 8:1621-1633.

440 [21] Norris P, Noble M, Francolini I, Vinogradov AM, Stewart PS, Ratner BD, Stoodley P. 441 Ultrasonically controlled release of ciprofloxacin from self-assembled coatings on poly (2-hydroxyethyl 442 methacrylate) hydrogels for Pseudomonas aeruginosa biofilm prevention. Antimicrob Agents Chemother $443 \quad 2005 ; 49(10): 4272-4279$. 
444 [22] Lentacker I, De Geest BG, Vandenbroucke RE, Peeters L, Demeester J, De Smedt SC, Sanders N. 445 Ultrasound-responsive polymer-coated microbubbles that bind and protect DNA. Langmuir 2006;22(17): $446 \quad 7273-7278$.

447 [23] Marmottant P, \& Hilgenfeldt S. Controlled vesicle deformation and lysis by single oscillating 448 bubbles, Nature 2003;423(6936): 153-156.

449 [24] Kost J, Leong K, Langer R. Ultrasound-enhanced polymer degradation and release of incorporated $450 \quad$ substances. Proc Natl Acad Sci U.S.A 1989; 86(20):7663-7666.

451 [25] Kost J, Leong K, Langer R. Ultrasonic modulated drug delivery systems. In Polymers in Medicine 452 II, Springer ; 1986, p. 387-396.

453 [26] Lavon I, Kost J. Mass transport enhancement by ultrasound in non-degradable polymeric controlled 454 release systems. J Control Release 1998; 54(1): 1-7.

455 [27] Liu LS, Kost J, D'Emanuele A, Langer R. Experimental approach to elucidate the mechanism of 456 ultrasound-enhanced polymer erosion and release of incorporated substances. Macromolecules 1992; $457 \quad 25(1): 123-128$.

458 [28] Kost J, Leong K, Langer R. Ultrasonically controlled polymeric drug delivery. In: Macromolecular 459 Symposia, Hüthig \& Wepf Verlag; 1998, p. 275-285 .

460 [29] Kost J, Liu LS, Gabelnick H, Langer R. Ultrasound as a potential trigger to terminate the activity of 461 contraceptive delivery implants. J Control Release 1994; 30(1): 77-81.

462 [30] Miyazaki S, Yokouchi C, Takada M. External control of drug release: controlled release of insulin 463 from a hydrophilic polymer implant by ultrasound irradiation in diabetic rats. J Pharm Pharmacol 464 1998;40(10): 716-717.

465 [31]Miyazaki S, Hou WM, Takada M. Controlled drug release by ultrasound irradiation. Chem Pharm 466 Bull 1985; 33(1): 428-431. 
467 [32] Chen F, Ma M, Wang J, Wang F, Chern SX, Zhao ER, Jhunjhunwala A, Darmadi S, Chen H, 468 Jokerst JV. Exosome-like silica nanoparticles: a novel ultrasound contrast agent for stem cell imaging. $469 \quad$ Nanoscale 2017; 9 (1): 402-411.

470 [33] Chen F, Zhao E, Kim T, Wang J, Hableel J, Reardon P, Ananthakrishna SJ, Wang T, Arconada471 Alvarez S, Knowles JC and Jokerst JV. Organosilica Nanoparticles with an Intrinsic Secondary Amine: 472 An Efficient and Reusable Adsorbent for Dyes. ACS Appl. Mater. Interfaces, 2017, 9 (18):15566-15576.

473 [34] El Fagui A, Dalmas F, Lorthioir C, Wintgens V, Volet G, Amiel C. Well-defined core-shell 474 nanoparticles containing cyclodextrin in the shell: a comprehensive study. Polymer 2011; 52(17): 37524753761.

476 [35] Grabar KC, Freeman RG, Hommer MB, Natan MJ. Preparation and Characterization of Au Colloid 477 Monolayers. Anal Chem 1995; 67: 735-743.

478

479

480

481

482

483

484

485

486

487

488

489

[36] Apfel RE, Holland CK. Gauging the likelihood of cavitation from short-pulse, low-duty cycle diagnostic ultrasound. Ultrasound Med Biol 1991;17(2): 179-185.

[37] Hendee WR, Ritenour ER. Medical imaging physics. John Wiley \& Sons; 2003.

[38] El Fagui A, Wintgens V, Gaillet C, Dubot P, Amiel C. Layer-by-Layer Coated PLA Nanoparticles with Oppositely Charged $\beta$-Cyclodextrin Polymer for Controlled Delivery of Lipophilic Molecules. Macromol Chem Phys 2014; 215(6): 555-565.

[39] Dubreuil F, Shchukin DG, Sukhorukov GB, Fery A. Polyelectrolyte capsules modified with YF3 nanoparticles: an AFM study. Macromol Rapid Co 2004; 25(11):1078-1081.

[40] Rae J, Ashokkumar M, Eulaerts O, von Sonntag C, Reisse J, Grieser F. Estimation of ultrasound induced cavitation bubble temperatures in aqueous solutions. Ultrason Sonochem 2005; 12(5): 325-329.

[41] Shchukin DG, Gorin DA, Möhwald H. Ultrasonically induced opening of polyelectrolyte microcontainers. Langmuir 2006; 22(17):7400-740. 
490 [42] Skirtach AG, De Geest BG, Mamedov A, Antipov AA, Kotov NA, Sukhorukov GB. Ultrasound 491 stimulated release and catalysis using polyelectrolyte multilayer capsules. J Mater Chem 2007; $492 \quad 17(11): 1050-1054$.

493 [43] De Geest BG, Skirtach AG, Mamedov AA, Antipov AA, Kotov NA, De Smedt SC, Sukhorukov 494 GB. Ultrasound-Triggered Release from Multilayered Capsules. Small 2007; 3(5): 804-808.

495 [44] Kolesnikova TA, Gorin DA, Fernandes P, Kessel S, Khomutov GB, Fery A, Möhwald H. 496 Nanocomposite microcontainers with high ultrasound sensitivity. Adv Funct Mater 2010;20(7):1189$497 \quad 1195$.

498 [45] Skirtach AG, Dejugnat C, Braun D, Susha AS, Rogach AL, Parak WJ, Sukhorukov G B. The role of 499 metal nanoparticles in remote release of encapsulated materials. Nano Lett 2005; 5(7): 1371-1377. 